ఠ

CASE REPORT

\title{
Advanced lung adenocarcinoma with spinal cord metastasis successfully treated with second EGFR-TKI treatment: a case report and literature review
}

\author{
This article was published in the following Dove Press journal: \\ OncoTargets and Therapy \\ 29 September 2015 \\ Number of times this article has been viewed
}

\section{Xiaoyan Li \\ Hui Yang \\ Jing Zhao \\ Hongjun Gao \\ Department of Lung Cancer, Affiliated Hospital of Academy of Military Medical Sciences, Fengtai District, Beijing, People's Republic of China}

Correspondence: Hongjun Gao Department of Lung Cancer, Affiliated Hospital of Academy of Military Medical Sciences, No 8 Dongdajie Street,

Fengtai District, Beijing 10007I, People's Republic of China

Tel +861066947161

Fax +86 I0 5III28605

Email gaohj6708@hotmail.com

\begin{abstract}
Lung adenocarcinoma can be accompanied by epidermal growth factor receptor (EGFR) mutation. As a consequence, targeted therapy based on screening of tyrosine-kinase inhibitors (TKIs) is necessary. Spinal cord metastasis of lung cancer is a serious clinical problem. Here, we report a case of lung adenocarcinoma with severe spinal cord metastasis that was successfully treated with a second administration of an EGFR-TKI. Magnetic resonance imaging showed spinal cord metastasis to the cervical vertebrae. The patient received a second administration of an EGFR-TKI (150 mg/day erlotinib). Four months later, we observed the disappearance of the paravertebral tumor and a reduction in the size of pulmonary tumors in both lungs. These results indicate that EGFR-TKI therapy could be a new strategy for the treatment of advanced lung adenocarcinoma with spinal metastasis.
\end{abstract}

Keywords: erlotinib, target therapy, NSCLC, compression of spinal cord

\section{Introduction}

Lung cancer is one of the main causes of cancer-related mortality in the People's Republic of China. The mortality rate for lung cancer is 45.57 per 100,000 patients and is 1.94 times higher in males $(61.00$ per 100,000$)$ than in females $(29.77$ per 100,000$) .{ }^{1}$ Lung cancer can metastasize to other organs such as the bone, adrenal gland, and brain, and metastasis to the spinal cord is an especially serious clinical problem. The etiology of spinal cord metastasis of lung cancer remains unclear. The incidence of spinal cord metastasis is low, but the prognosis is poor. ${ }^{2}$ Magnetic resonance imaging (MRI) is necessary for first-line examination, and computed tomography (CT) scans are helpful at some stages such as diagnosis and postoperative follow-up of spinal metastatic disease. ${ }^{3}$

Lung adenocarcinoma can be accompanied by epidermal growth factor receptor (EGFR) mutation. As a consequence, targeted therapy based on screening of tyrosinekinase inhibitors (TKIs) is necessary. Treatment with an EGFR-TKI, such as gefitinib or erlotinib, is an effective targeting therapy, particularly for advanced non-small-cell lung cancer (NSCLC). EGFR-TKI treatment has been demonstrated to significantly improve responses and outcomes in patients with advanced NSCLC harboring an EGFR mutation. ${ }^{1}$

Interestingly, in some patients with lung cancer who were negative for EGFR, it has been reported that EGFR-TKIs exhibit superior effects over conventional chemotherapies. Notably, the patient characteristics of being Asian, having an adenocarcinoma, being 
female, and being a nonsmoker are regarded as favorable predictors for EGFR-TKI efficacy in NSCLC with unknown EGFR gene status. ${ }^{4}$ Some of these patients were also found to benefit from the second administration of EGFR-TKI. However, the benefits of EGFR-TKI therapy against spinal cord metastasis of lung cancer remain unclear. Here, we report a case of lung adenocarcinoma with severe spinal cord metastasis that was successfully treated via a second administration of a TKI, and we discuss the benefits of repeated EGFR-TKI therapy as a new treatment strategy for spinal cord metastasis.

\section{Case report}

A 39-year-old female presented with reduced muscle strength in the right upper limb was admitted to our hospital in April 2011. Cerebral MRI showed encephalic multiple foci, indicating the possibility of a metastatic tumor. According to the chest CT scan, the patient was diagnosed with right lung carcinoma accompanied by metastases to the mediastinum lymph nodes, both lungs, bone, and brain.

The patient underwent a needle biopsy of the inferior pulmonary focus under CT scanning, and pathological diagnosis confirmed that she had adenocarcinoma. Nevertheless, we could not perform an EGFR mutation test due to the limited size of samples. Considering all the examination data and images, the stage of lung adenocarcinoma in this case was diagnosed as $\mathrm{T}_{4} \mathrm{~N}_{2} \mathrm{M}_{1}$ (stage IV).

The patient refused chemotherapy. Considering that the patient had favorable predictor factors for EGFR-TKI efficacy in NSCLC with unknown EGFR gene status, ${ }^{4}$ such as being Asian, having an adenocarcinoma, being female, and being a nonsmoker, the patient received first-line treatment with $250 \mathrm{mg}$ /day gefitinib starting March 1, 2011. Partial response (PR) was detected, and progression-free survival (PFS) lasted for 14 months (Figure 1). In addition, she received wholebrain radiation therapy with Dt40Gy/20f starting March 3,
2011. From June 22, 2012 to November 27, 2012, the patient received second-line chemotherapy with six cycles of a cisplatin and pemetrexed regimen. Next, she received two cycles of pemetrexed chemotherapy, and the best response was stable disease with PFS lasting for 8 months. As the disease was not improved significantly, she received docetaxel combined with carboplatin for four cycles with the best response of stable disease and PFS of only 3.5 months. Then, the patient was treated with carboplatin plus gemcitabine as fourth-line therapy for two cycles with the result of progressive disease.

At the end of September 2013, the patient's condition had deteriorated significantly. She had difficulty of moving both lower limbs, especially the right lower limb, gradually leading to an incomplete paralysis. Cervical vertebral MRI showed a metastatic tumor in the cervical vertebral canal that compressed the spinal cord at the second cervical level. After multidisciplinary consultation, the patient refused treatment with surgery and local radiation therapy. Therefore, we chose erlotinib as the fifth-line therapy at the dose of $150 \mathrm{mg} /$ day starting October 10, 2013. After the second administration of an EGFR-TKI, the paraspinal tumor disappeared (Figure 2), and tumors in both lungs shrank significantly (Figure 3). The objective response was PR. Additionally, the muscle strength in both upper limbs recovered to degree IV, and that in the lower limbs recovered to degree I after 1 week of erlotinib treatment. After 4 months of erlotinib treatment, the muscle strength had recovered to degree $\mathrm{V}$ in the upper limbs and to degrees II and III in the lower limbs.

Because the patient refused a second puncture biopsy, a plasma sample was taken for EGFR mutation detection using the amplification refractory mutation system (ARMS) method on February 28, 2014, and the result was negative. According to the latest follow-up on August 17, 2014, the patient continued taking erlotinib orally.


Figure I Computed tomography scan of the lung before and after 14 months of gefitinib treatment. 

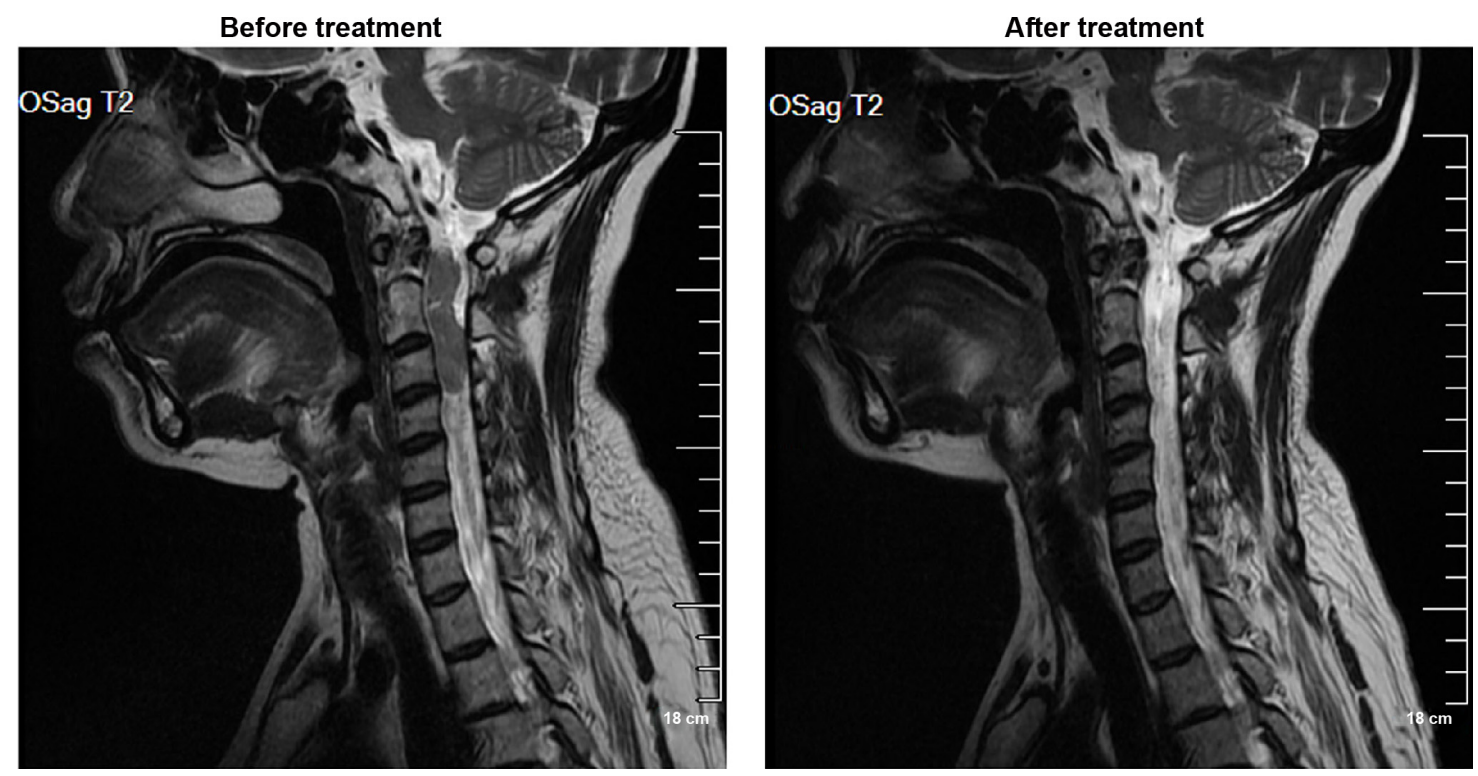

Figure 2 Magnetic resonance imaging showing the disappearance of the paraspinal tumor after erlotinib treatment.

Written informed consent was obtained from the patient according to the Declaration of Helsinki, and the study protocol was approved by the research ethics committee of the Affiliated Hospital of Academy of Military Medical Sciences, Beijing, People's Republic of China.

\section{Discussion}

In this case, the patient had received gefitinib and chemotherapy previously. However, after nearly 3 years of treatment, spinal cord metastasis was found and the prognosis was very poor. Because there were no more treatment options to choose and considering the poor performance, the patient was given oral erlotinib, as the second administration of another EGFRTKI might experience an unexpected PR.5,6 This outcome demonstrated that the administration of a TKI could be a new effective method in treating lung cancer with spinal cord metastasis. In addition, we reviewed the literature related to EGFR-TKI therapy for lung adenocarcinoma and postulated the possible reasons for the satisfactory therapeutic effects of a TKI for lung cancer with spinal cord metastasis.

Conventional treatment for spinal cord metastasis is tumor resection, which may be effective but not thorough enough, and therefore, cases of recurrence are common. Other therapies include irradiation and chemotherapy, and chemotherapy penetrates poorly to the central nervous system (CNS) with potentially low efficiency for these patients. So far, a common management for lung cancer metastasis is stereotactic radiotherapy at the 60 Gy conventional fractions in patients with NSCLC. Nevertheless, the associated neurological symptoms and outcomes for patients with symptomatic vertebral compression are still in debate. ${ }^{7}$ Recent case reports document that EGFR-TKI may be a good option for the
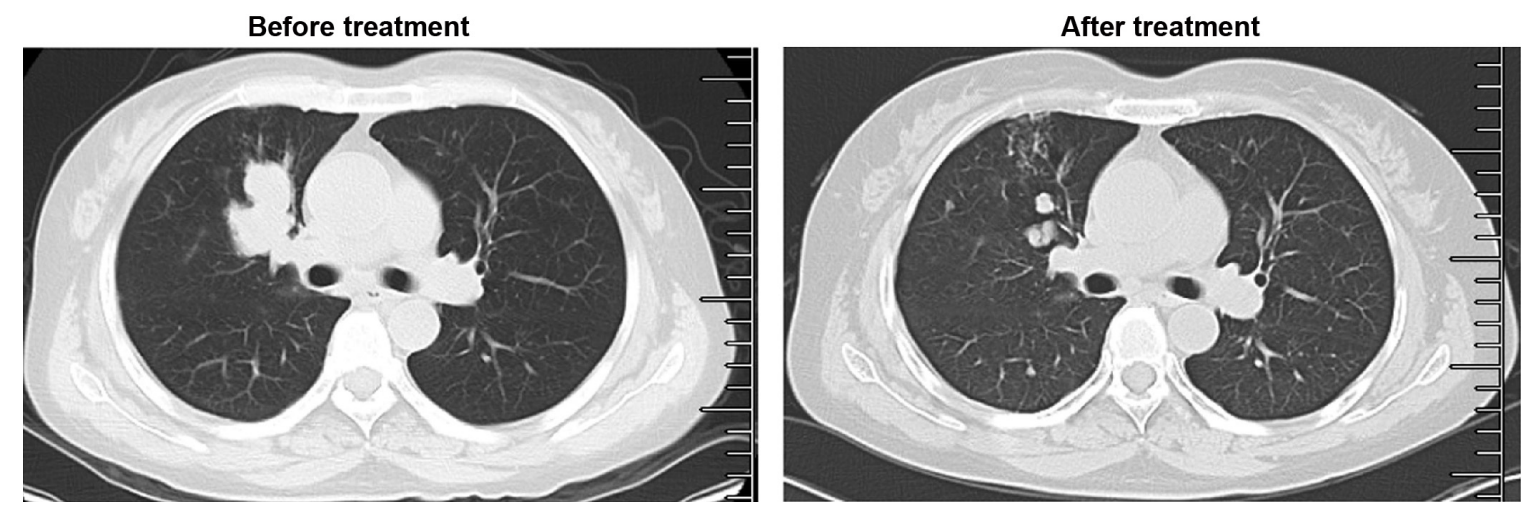

Figure 3 Computed tomography scan showing that the lung tumor shrank significantly after erlotinib treatment. 
treatment of CNS metastasis of lung cancer patients with EGFR mutations. Xu et al and Zhang et al recently reported that EGFR mutation-positive lung cancer patients with CNS metastasis were successfully treated with either erlotinib or icotinib singly or in combination with surgery/irradiation. ${ }^{8,9}$ Although we cannot obtain the evidence of EGFR mutation directly from metastatic spinal cord tumor of our reported patient, we chose a second administration of erlotinib instead of resection or continued chemotherapy for the treatment of her metastasis considering that the first administration of TKI was effective and surgery and radiotherapy were not suitable for spinal cord metastasis of lung cancer; consistent with recent reports, ${ }^{8,9}$ we observed that paraspinal tumor disappeared and tumors in both lungs shrank significantly in our case with final outcome defined as PR. The outcomes in this case indicate that the application of an EGFR-TKI could be a new treatment method for spinal cord metastasis. TKIs have additional advantages of penetrating to the central nerve system. It has been reported that erlotinib reaches higher concentration in cerebrospinal fluid than gefitinib, and a high cerebrospinal fluid concentration of erlotinib depends on a high plasma concentration. ${ }^{10}$ In addition, accumulation of erlotinib at sites of metastasis was detected by an isotopic tracer. ${ }^{11}$ Therefore, the high concentration of erlotinib and its accumulation in sites of metastasis could be responsible for the satisfactory outcome in this case.

Recently, TKIs have been proposed as an important therapy for NSCLC, and EGFR mutation analysis has become a routine clinical test. Almost 20 EGFR mutations have been detected, direct sequencing is commonly used for the detection of an EGFR mutation, but its rather low sensitivity has led to the development of more sensitive methods, including real-time polymerase chain reaction and ARMS. ${ }^{2}$

It is well-known that DNA from tumor tissue is the best material for EGFR mutation analysis. Unfortunately, most patients with NSCLC are at the advanced stage and inoperable, thus making collection of a sufficient amount of tumor tissue difficult. In contrast, collection of a body fluid such as plasma is easy, less invasive, and repeatable. Moreover, body fluid is considered a feasible and reliable source for genomic DNA. In this case, we performed ARMS to detect EGFR mutation in a plasma sample and obtained negative results. A previous study compared the results of ARMS and direct sequencing for the detection of an EGFR mutation and prediction of EGFR-TKI efficacy for surgery and biopsy tumor tissues in 158 patients with NSCLC, ${ }^{12}$ which showed that ARMS has a higher sensitivity and specificity than direct sequencing for the detection of an EGFR mutation in both surgical and biopsy samples. Moreover, the results from ARMS are more consistent with the efficacy of EGFRTKI treatment. ${ }^{12}$ In addition, several studies have shown that serum samples can be an alternative source for mutation analysis, and a high correlation was found between mutations detected in serum samples and matched tumor samples. ${ }^{13,14}$ Taken together, these results indicate that plasma/serum samples could be an attractive source for the detection of an EGFR mutation and TKI sensitivity. Nevertheless, although the sensitivity of ARMS is high, if the abundance of the DNA mutation is below the limit, a false-negative result may occur. Therefore, in the present case, we could not exclude the possibility of a false negative, especially considering that our patient benefited from a second round of TKI therapy. Further confirmation of our results based on tumor tissue is necessary.

A certain sensitive mutation of EGFR is considered to be the best predictor for the clinical benefits of chemotherapy. However, patients without a feasible mutation could be identified as "dominant people" or the opposite on the basis of certain clinical characteristics. For example, smoking status (nonsmoker), histology (adenocarcinoma/bronchioloalveolar carcinoma), and sex (female) could cause a higher possibility of mutation and result in a longer survival after erlotinib treatment. ${ }^{1}$ EGFR mutant lung cancer represents a distinct subgroup for TKI treatment, and chemotherapy is recommended as the first-line therapy for advanced unselected patients with NSCLC. Due to inadequate tissue samples, we were not able to detect an EGFR mutation before treatment. In order to identify patients for whom treatment with erlotinib and gefitinib is suitable, we must collect adequate biopsy samples to detect EGFR mutations and ensure the success of personalized therapy in future clinical studies. ${ }^{3}$

It has been reported that patients could benefit from retreatment with an EGFR-TKI if the first treatment with erlotinib therapy was effective. Song et al investigated the efficacy of re-administration of a TKI after failure of gefitinib or erlotinib in 33 patients with NSCLC and showed that re-administration of TKI seemed to be a potential therapeutic option for the treatment of selected advanced patients with NSCLC after prior failure, especially for the patients who responded to the first TKI treatment. ${ }^{15}$ Moreover, Tang et al re-administrated EGFR-TKI to 120 patients with NSCLC and found that a time interval of $\geq 4$ months might improve the treatment, but differences in clinical benefit were not apparent among the different treatment orders. ${ }^{16}$ These data suggest that patients who benefit from prior treatment may be more likely to benefit from a second administration. 
In the present case, the patient was given EGFR-TKI treatment using gefitinib followed by erlotinib, and the time interval was $>2$ years. Because the patient had benefited from the first administration of this treatment, and the order of treatment we followed had been proven effective in previous studies, we hypothesized that a second administration of erlotinib was an appropriate option. The outcomes of this case indicate that EGFR-TKI therapy could be a new strategy for the treatment of spinal metastasis, and such benefit is related to the PFS following the initial TKI therapy and the time interval between the two applications of the TKI.

\section{Disclosure}

The authors report no conflicts of interest in this work.

\section{References}

1. Li W, Zhou F, Zhou C. Role of erlotinib in the targeted treatment of non-small-cell lung cancer in Chinese patients. OncoTargets Ther. 2014; 7:253-261.

2. Hata Y, Takai Y, Takahashi H, et al. Complete response of 7 years' duration after chemoradiotherapy followed by gefitinib in a patient with intramedullary spinal cord metastasis from lung adenocarcinoma. J Thorac Dis. 2013;5(2):E65-E67.

3. Shah LM, Salzman KL. Imaging of spinal metastatic disease. Int J Surg Oncol. 2011;2011:769753.

4. Zeng Z, Chen HJ, Yan HH, Yang JJ, Zhang XC, Wu YL. Sensitivity to epidermal growth factor receptor tyrosine kinase inhibitors in males, smokers, and non-adenocarcinoma lung cancer in patients with EGFR mutations. Int J Biol Markers. 2013;28(3):249-258.

5. Cho BC, Im CK, Park MS, et al. Phase II study of erlotinib in advanced non-small-cell lung cancer after failure of gefitinib. J Clin Oncol. 2007; 25(18):2528-2533.
6. Lee DH, Kim SW, Suh C, Yoon DH, Yi EJ, Lee JS. Phase II study of erlotinib as a salvage treatment for non-small-cell lung cancer patients after failure of gefitinib treatment. Ann Oncol. 2008;19(12):2039-2042.

7. Lee DS, Kwak YK, Jeong SM, et al. High-dose radiotherapy using helical tomotherapy for vertebral metastasis: early clinical outcomes and cord dose specification. Jpn J Clin Oncol. 2013;43(6):646-653.

8. Xu S, Yu X, Xu M. Long-term survival of a patient with lung cancer metastasis to the spine following surgical treatment combined with radiation and epithelial growth factor receptor inhibitor therapy: a case report. Exp Ther Med. 2015;9(1):117-119.

9. Zhang Y, Tang H, Li J, Li M. An active treatment of lung adenocarcinoma cancer with brain metastases: icotinib. OncoTargets Ther. 2015; 8:1351-1354.

10. Togashi Y, Masago K, Fukudo M, et al. Efficacy of increased-dose erlotinib for central nervous system metastases in non-small cell lung cancer patients with epidermal growth factor receptor mutation. Cancer Chemother Pharmacol. 2011;68(4):1089-1092.

11. Weber B, Winterdahl M, Memon A, et al. Erlotinib accumulation in brain metastases from non-small cell lung cancer: visualization by positron emission tomography in a patient harboring a mutation in the epidermal growth factor receptor. $J$ Thorac Oncol. 2011;6(7):1287-1289.

12. Shaozhang Z, Ming Z, Haiyan P, Aiping Z, Qitao Y, Xiangqun S. Comparison of ARMS and direct sequencing for detection of EGFR mutation and prediction of EGFR-TKI efficacy between surgery and biopsy tumor tissues in NSCLC patients. Med Oncol. 2014;31(5):926.

13. Akca H, Demiray A, Yaren A, et al. Utility of serum DNA and pyrosequencing for the detection of EGFR mutations in non-small cell lung cancer. Cancer Genet. 2013;206(3):73-80.

14. Kim ST, Jung HY, Sung JS, et al. Can serum be used for analyzing the EGFR mutation status in patients with advanced non-small cell lung cancer? Am J Clin Oncol. 2013;36(1):57-63.

15. Song Z, Yu X, He C, Zhang B, Zhang Y. Re-administration after the failure of gefitinib or erlotinib in patients with advanced non-small cell lung cancer. J Thorac Dis. 2013;5(4):400-405.

16. Tang $\mathrm{C}, \mathrm{Gao} \mathrm{H}, \mathrm{Li}$ X, et al. Different treatment orders achieved similar clinical results: a retrospective study for retreatment of epidermal growth factor receptor tyrosine kinase inhibitors in 120 patients with non-smallcell lung cancer. J Cancer Res Clin Oncol. 2014;140(3):427-433.
OncoTargets and Therapy

\section{Publish your work in this journal}

OncoTargets and Therapy is an international, peer-reviewed, open access journal focusing on the pathological basis of all cancers, potential targets for therapy and treatment protocols employed to improve the management of cancer patients. The journal also focuses on the impact of management programs and new therapeutic agents and protocols on

\section{Dovepress}

patient perspectives such as quality of life, adherence and satisfaction. The manuscript management system is completely online and includes a very quick and fair peer-review system, which is all easy to use. Visit http://www.dovepress.com/testimonials.php to read real quotes from published authors. 